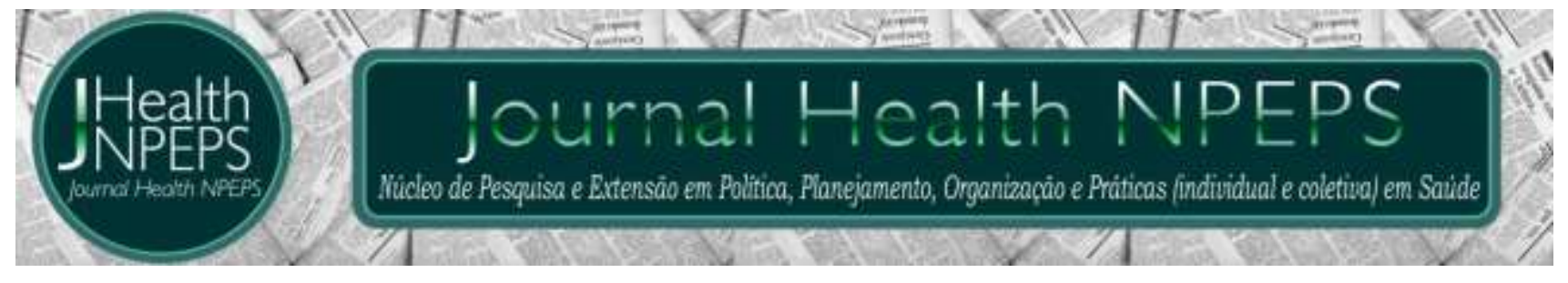

http://dx.doi.org/10.30681/252610104844

ARTIGO ORIGINAL

\title{
Epidemiologia da COVID-19 em Uberlândia (MG): análise preliminar do impacto do grau de abertura comercial
}

\section{Epidemiology of COVID-19 in Uberlândia (MG): preliminary analysis of the impact of commercial activities's openness}

\section{Epidemiología del COVID-19 en Uberlândia (MG): análisis preliminar del impacto del grado de apertura comercial}

\section{Maria Fernanda Prado Rosa ${ }^{1}$, William Nicoleti Turazza da Silva², Wellington Roberto Gomes de Carvalho ${ }^{3}$, Stefan Vilges de Oliveira ${ }^{4}$}

\section{RESUMO}

Objetivo: analisar os dados epidemiológicos da COVID-19 em Uberlândia e confrontá-los com as determinações municipais de abertura e fechamento comercial em diferentes momentos da pandemia. Método: trata-se de estudo epidemiológico, observacional e descritivo, utilizando dados secundários, referente aos casos notificados da COVID-19 em Uberlândia. Resultados: foram realizados 59.994 testes, sendo 14.389 positivos $(23,98 \%)$ e 45.605 negativos (76,02\%). Registraram-se 265 óbitos e taxa de letalidade de 1,89\%. Casos confirmados apresentaram tendência de crescimento após abertura comercial, tendência de estabilização após fechamento comercial, e tendência de queda após a última reabertura comercial, acompanhada de acúmulo de testes suspeitos e queda da testagem populacional. Entre os internados diários, houve predomínio de homens (59\%), maiores de 60 anos (55\%) e alocados em leitos de enfermaria (55\%). Conclusão: percebeu-se expressiva influência do grau de abertura comercial nos indicadores da doença, com incremento da frequência de testes positivos e óbitos, e manutenção das internações.

Drescritores: Infecções por Coronavirus; Epidemiologia; Pandemias; Monitoramento Epidemiológico; Vigilância em Saúde Pública.

\footnotetext{
${ }^{1}$ Graduanda em Medicina pela Universidade Federal de Uberlândia (UFU). Uberlândia, Minas Gerais, Brasil. E-mail: prosananda@gmail.com ORCID ID: https://orcid.org/0000-0001-5067-7894

${ }^{2}$ Graduando em Medicina pela Universidade Federal de Uberlândia (UFU). Uberlândia, Minas Gerais, Brasil. E-mail: williamnicolete@gmail.com ORCID ID: https://orcid.org/0000-0001-6942-992X

${ }^{3}$ Educador físico. Doutor em Saúde da Criança e do Adolescente. Professor Adjunto. Instituto de Geografia, Curso de Saúde Coletiva, Universidade Federal de Uberlândia (UFU). Uberlândia, Minas Gerais, Brasil. E-mail: wrgcarvalho@ufu.br ORCID ID: https://orcid.org/0000-0003-4185-526X

${ }^{4}$ Biólogo. Doutor em Medicina Tropical. Professor Adjunto do Departamento de Saúde Coletiva, Faculdade de Medicina, Universidade Federal de Uberlândia (UFU). Uberlândia, Minas Gerais, Brasil. E-mail: stefan@ufu.br ORCID ID: https://orcid.org/0000-0002-5493-2765 Autor para Correspondência - Endereço: Departamento de Saúde Coletiva. Avenida Pará, 1720, Campus Umuarama, Bloco 2U, Sala 8, Umuarama, CEP 38405320. Uberlândia, Minas Gerais, Brasil.
}

Este artigo está licenciado sob forma de uma licença Creative Commons Atribuição 4.0 Internacional, que permite uso irrestrito, distribuição e reprodução em qualquer meio, desde que a publicação original seja corretamente citada 


\begin{abstract}
Objective: to analyze COVID-19's epidemiological data in Uberlândia and compare them with the municipal determinations of opening and closing of commercial's acitivities at different times of the pandemic. Method: this is an epidemiological, observational and descriptive study, using secondary data, referring to notified cases of COVID-19 in Uberlândia. Results: 59,994 tests were performed, of which 14,389 were positive $(23.98 \%)$ and 45,605 negative $(76.02 \%)$. There were 265 deaths and a fatality rate of $1.89 \%$. Confirmed cases showed a growth tendency after commercial opening, a stabilization tendecy after commercial closing, and a downward tendecy after the last commercial reopening, accompanied by an accumulation of suspicious tests and a drop in population testing. Among daily inpatients, there was a predominance of men (59\%), older than 60 years old (55\%) and allocated to infirmary beds (55\%). Conclusion: there was a significant influence of the degree of commercial opening in the indicators of the disease, with an increase in the frequency of positive tests and deaths, and maintenance of hospitalizations
\end{abstract}

Descriptors: Coronavirus Infections; Epidemiology, Pandemics; Epidemiological Monitoring; Public Health Surveillance.

\title{
RESUMEN
}

Objetivo: analizar los datos epidemiológicos de COVID-19 en Uberlândia y confrontarlos con las determinaciones municipales de apertura y cierre comercial en diferentes momentos de la pandemia. Método: se trata de un estudio epidemiológico, observacional y descriptivo, utilizando datos secundarios, referidos a los casos notificados de COVID-19 en Uberlândia. Resultados: se realizaron 59.994 pruebas, de las cuales 14.389 fueron positivas (23,98\%) y 45,605 negativas (76,02\%). Hubo 265 muertes y una tasa de mortalidad del $1,89 \%$. Los casos confirmados mostraron una tendencia de crecimiento después de la apertura comercial, una tendencia de estabilización después del cierre comercial y una tendencia a la baja después de la última reapertura comercial, acompañada de una acumulación de pruebas sospechosas y una caída en las pruebas de población. Entre los internados diarios, hubo predominio de hombres (59\%), mayores de 60 años (55\%) y asignados a camas de enfermería (55\%). Conclusión: hubo una influencia significativa del grado de apertura comercial en los indicadores de la enfermedad, con un aumento en la frecuencia de pruebas positivas y muertes, y mantenimiento de hospitalizaciones.

Descriptores: Infecciones por Coronavirus; Epidemiología; Pandemias; Monitoreo Epidemiológico; Vigilancia en Salud Pública.

\section{INTRODUÇÃO}

Em 31 de Dezembro de 2019, a China reportou à Organização Mundial de Saúde (OMS) um grupo de indivíduos diagnosticados com uma espécie de pneumonia, sem etiologia definida, detectada na cidade de Wuhan ${ }^{1-4}$. Cerca de 12 dias depois, os chineses compartilharam o sequenciamento do novo vírus, o coronavírus da síndrome respiratória aguda grave 2 (SARS-CoV$2)^{2,4}$. No mesmo mês, a OMS convocou duas reuniões do Comitê de Emergência, sendo que na última constataram o crescimento do número de casos e de 
países afetados, decretando a situação como uma Emergência de Saúde Pública de Importância Internacional (ESPII) $)^{3,4}$. Já no Brasil, o primeiro caso da doença pelo Coronavírus (COVID-19) foi confirmado em 26 de fevereiro de 2020, na cidade de São Paulo ${ }^{4}$.

Segundo estudos, o tempo de médio de duplicação da doença varia entre 5,2 a 7,4 dias ${ }^{5-7}$. Dessa forma, a velocidade de progressão da epidemia é elevada. Diante desse potencial de transmissão da doença, juntamente com a ausência de medidas imediatas capazes de conter a infecção, como fármacos ou vacinas, as intervenções na estrutura social são medidas essenciais e urgentes. Nesse sentido, o isolamento social se mostrou a opção mais adequada para essas condições, principalmente pelo respaldo das experiências mundiais ${ }^{6}$. As medidas de distanciamento social visam evitar a superlotação do Sistema Único de Saúde (SUS) que ocorreria mediante a infecção simultânea e maciça, de forma a garantir o acesso adequado à população ao suporte ventilatório e ao cuidado em Unidades de Terapia Intensiva (UTI) ${ }^{5}$.

Entretanto, sabe-se que o impacto econômico causado pela pandemia e a paralisação de diversas atividades comerciais é grande, principalmente em países considerados subdesenvolvidos ou em desenvolvimento, com importantes consequências na qualidade de vida da população8-10. No Brasil, debate-se frequentemente a necessidade de adoção de períodos de maior flexibilização nas medidas de isolamento social, visando garantir fonte de renda e movimentação econômica no país`. Para que isso seja possível, torna-se indispensável a atuação efetiva da Vigilância Epidemiológica. 0 SUS preconiza que seja realizada essa vigilância, ou seja, ações orientadas no sentido de coleta efetiva de dados, com a finalidade de definir a dinâmica de uma doença ou agravo à saúde. Com as variáveis coletadas, há produção e a divulgação de informações epidemiológicas as quais servem para guiar a gestão dos diversos serviços e níveis de atenção ${ }^{11,12}$.

No cenário da pandemia, esse monitoramento epidemiológico desempenha papel fundamental no enfrentamento da doença, visto que a análise dos dados permite a constatação dos padrões referentes a COVID-19 de forma a prever seu comportamento, bem como sua evolução. Dessa maneira, são geradas informações importantes para direcionamento racional das políticas 
públicas, no sentido de se estabelecer um planejamento estratégico para o efetivo controle da pandemia ${ }^{5,11,12}$.

No contexto das medidas de vigilância em saúde, no dia 27 de fevereiro de 2020, foi decretado o surgimento do Comitê Municipal de Enfrentamento a COVID-19 de Uberlândia, a fim de instituir ações preventivas em relação ao novo coronavírus $^{13}$. Dessa forma, o presente estudo tem como objetivo analisar o perfil epidemiológico da COVID-19 em Uberlândia e confrontá-lo com as ações executivas, desempenhadas pela prefeitura municipal, que propuseram aberturas e fechamentos do comércio em diferentes momentos durante a pandemia.

\section{MÉTODO}

$$
\text { Trata-se de estudo }
$$
epidemiológico, observacional e descritivo, utilizando dados secundários, dos casos notificados da COVID-19 à Secretaria Municipal de Saúde de Uberlândia (MG). Todos os registros foram obtidos por meio do Boletim Informativo Municipal de Uberlândia ${ }^{14}$. Os dados analisados referem-se aos casos notificados no período de 21 de março a 7 de agosto de 2020, totalizando 20 semanas. Nesse estudo, foram incluídos os casos que atenderam aos critérios diagnósticos para a COVID-19, de acordo com as diretrizes do Ministério da Saúde ${ }^{15}$.

O município de Uberlândia localiza-se na região Sudeste do Brasil, a oeste da capital do estado, Belo Horizonte. De acordo com o Instituto Brasileiro de Geografia e Estatística (IBGE) $^{16}$, o município possui uma população de 691.305 habitantes com uma densidade demográfica de 146,78 $\mathrm{hab} / \mathrm{km}^{2}$ e um Índice de Desenvolvimento Humano Municipal (IDHM) de 0,789.

As variáveis analisadas foram: número de casos e óbitos confirmados; número de casos e óbitos suspeitos; número de casos descartados; número de testes; número total de pacientes internados (em UTI e enfermaria); sexo biológico (masculino e feminino) e faixa etária (0-5, 6-12, 13-39, 40-59, 60-69, 70-79 e 80+anos).

Os dados apresentados de sexo, faixa etária e tipo de leito ocupado refletem apenas os dados diários, não havendo soma final da quantidade de indivíduos. Para o cálculo do percentual aproximado por sexo dos pacientes internados, realizou-se a soma das internações diárias do sexo em análise/ 
(soma das internações diárias do sexo masculino + soma das internações diárias do sexo feminino). Para o cálculo do percentual aproximado por tipo de leito ocupado pelos pacientes internados, somou-se as internações diárias do leito em análise/ (soma das internações diárias em leitos de UTI + soma das internações diárias em leitos de enfermaria). Para o cálculo do percentual aproximado por faixa etária dos pacientes internados, calculou-se as internações diárias da faixa etária em análise/ (soma das internações diárias de todas as faixas etárias). Portanto, os dados apresentados não refletem a quantidade final de indivíduos internados para cada variável analisada, somente uma estimativa, segundo a informação diária disponibilizada.

Para caracterizar a COVID-19, foi realizada uma análise epidemiológica por meio da estatística descritiva (frequências absolutas e relativas). A incidência dos casos da COVID-19 foi calculada utilizando o número de casos confirmados no município/população total x 100 mil habitantes. A mortalidade dos casos da COVID-19 foi calculada pelo número de óbitos confirmados no município/população total x $100 \mathrm{mil}$ habitantes. Para o calcular a letalidade da COVID-19, utilizou-se o número de óbitos pela doença/número total de casos confirmados $\times$ 100. A taxa de testagem da população foi calculada por meio do total de testes realizados/população total $x$ 100.000. 0 cálculo da média móvel foi realizado com base na soma do número de casos e /ou óbitos confirmados nos últimos sete dias, dividida por sete. Para estimar a taxa de crescimento diário da doença, dividiu-se o número total acumulado de casos confirmados do dia pelo número de total acumulado de casos confirmados do dia anterior.

Para avaliar o impacto preliminar da abertura e do fechamento do comércio, foram considerados três decretos do Comitê Municipal de Enfrentamento a COVID-19 da Prefeitura Municipal de Uberlândia. 0 primeiro foi deliberado em 15 de Abril de 2020 e dispôs sobre a reabertura das atividades comerciais no município ${ }^{17}$. O segundo, foi deliberado no dia 22 de Maio de 2020 e dispunha sobre a reabertura dos centros de lazer e de formação de condutores, entrando em vigor a partir do dia 28 de Maio de $2020^{18}$. O terceiro, foi deliberado no dia 19 de junho de 2020 determinando um novo fechamento das atividades comerciais, dos centros de lazer e de formação de condutores ${ }^{19}$. Em 17 de julho de 2020, foi estabelecida 
uma nova abertura comercial, que se estendeu até o término do período analisado ${ }^{20}$. A avaliação da tendência de crescimento, queda ou estabilização nos casos ocorreu a partir do monitoramento da média móvel, adotada globalmente no monitoramento de casos da COVID$19^{21}, 14$ dias após os decretos municipais: para aumento de casos superior a 15\%, considerou-se tendência de crescimento dos casos, para redução de casos superior a $>15 \%$ considerou-se tendência de queda nos casos e para aumentos ou reduções de $15 \%$ ou menos, considerou-se tendência de estabilização do número de casos. Os parâmetros pautam-se em critérios adotados pelo Consórcio de Veículos de Comunicação do Brasil22, que assumiu papel central na divulgação dos dados da pandemia frente à redução da divulgação de dados pelo Ministério da Saúde 23 .

Além disso, foram comparadas as médias das taxas de crescimento registradas nos 14 dias anteriores aos decretos municipais e nos 14 dias posteriores aos mesmos decretos, por média aritmética simples. Todas as análises foram realizadas pelo programa Microsoft Office Excel 2016.

0 presente estudo dispensou análise do Comitê de Ética em Pesquisa, por se tratar de dados secundários, de domínio e acesso público. Todos os dados utilizados encontram-se disponíveis para acesso e utilização pública no Boletim Municipal Diário divulgado pela Prefeitura Municipal de Uberlândia $^{14}$ e não possibilitam a identificação dos pacientes, estando de acordo com a Resolução n 466/2012 do Conselho Nacional de Saúde ${ }^{24}$ que estabelece normas e diretrizes regulamentadoras de pesquisas envolvendo seres humanos.

\section{RESULTADOS}

No período de 21 de março a 7 de agosto de 2020, foram realizados um total de 59.994 testes, desses 14.389 foram positivos $(23,98 \%)$ e 45.605 negativos (76,02\%). Nesse período, Uberlândia registou um total de 265 óbitos pela COVID-19. A incidência de casos confirmados da COVID-19 para o período foi de 2.081,42 casos/100 mil habitantes, a letalidade foi de 1,84\% e a taxa de mortalidade de 39,33 óbitos/100 mil habitantes. A taxa de testagem até o momento foi de $8.678,36$ testes $/ 100 \mathrm{mil}$ habitantes. Observou-se no período um aumento contínuo do número de pessoas testadas, de casos e de óbitos confirmados pela COVID-19 (Figura 1- A). 
A partir da segunda semana de maio/2020 houve crescimento importante da média móvel, principalmente para os casos confirmados pela doença, com períodos alternados de queda e crescimento (Figura 1 - B). Para os óbitos, também houve variação da média móvel, porém menor. As maiores taxas de crescimento da média móvel ocorreram principalmente na primeira metade do período analisado, havendo maior estabilização durante a segunda metade (Figura 1 - C).

Houve acúmulo inicial de casos suspeitos, atingindo pico de 5.954 em 16/05. Ao final do mês de julho, voltaram a ultrapassar a marca de dois mil casos, chegando aproximadamente a quatro mil casos no início de agosto, com indicativa subsequente de redução (Figura 2 - A). A variável “óbitos suspeitos" chegou até 10 uma única vez, sendo mais estável (Figura 2 - B). Houve crescimento importante da quantidade de testes por dia, acompanhada do aumento da média móvel, principalmente a partir da primeira semana de junho. Durante a última semana de julho, é possível observar diminuição expressiva na taxa de testagem diária da população (Figura 2 C).

Quando analisados os decretos municipais de abertura e fechamento comercial, notou-se tendência de crescimento da média móvel dos casos confirmados após 14 dias dos dois primeiros decretos municipais de maior abertura comercial, com estabilização após o único decreto de fechamento, e queda após o último decreto analisado, de reabertura comercial (Tabela 1). A média das taxas de crescimento apresentou comportamento semelhante ao das médias móveis, com crescimento percentual após as duas primeiras aberturas comerciais e redução tanto após o único decreto de fechamento comercial, quanto no último decreto de reabertura (Tabela 2 ). 


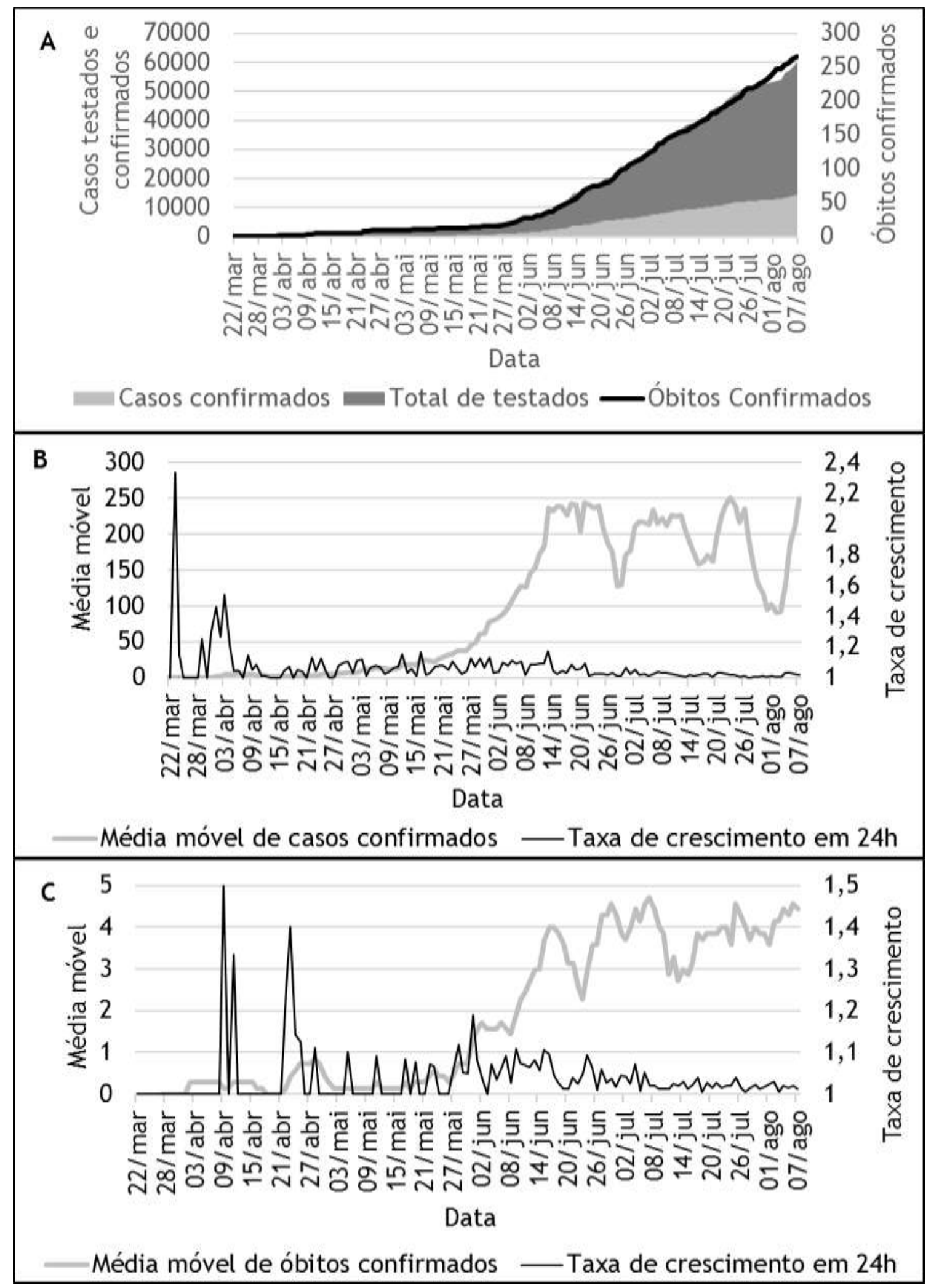

Figura 1 - A: Número total de indivíduos testados, casos e óbitos confirmados pela COVID19 em Uberlândia (MG); B: Média móvel e taxa de crescimento em 24 horas dos casos confirmados da COVID-19; C: Média móvel e taxa de crescimento em 24 horas dos óbitos confirmados pela COVID-19. Uberlândia (MG), Brasil, 2020. 


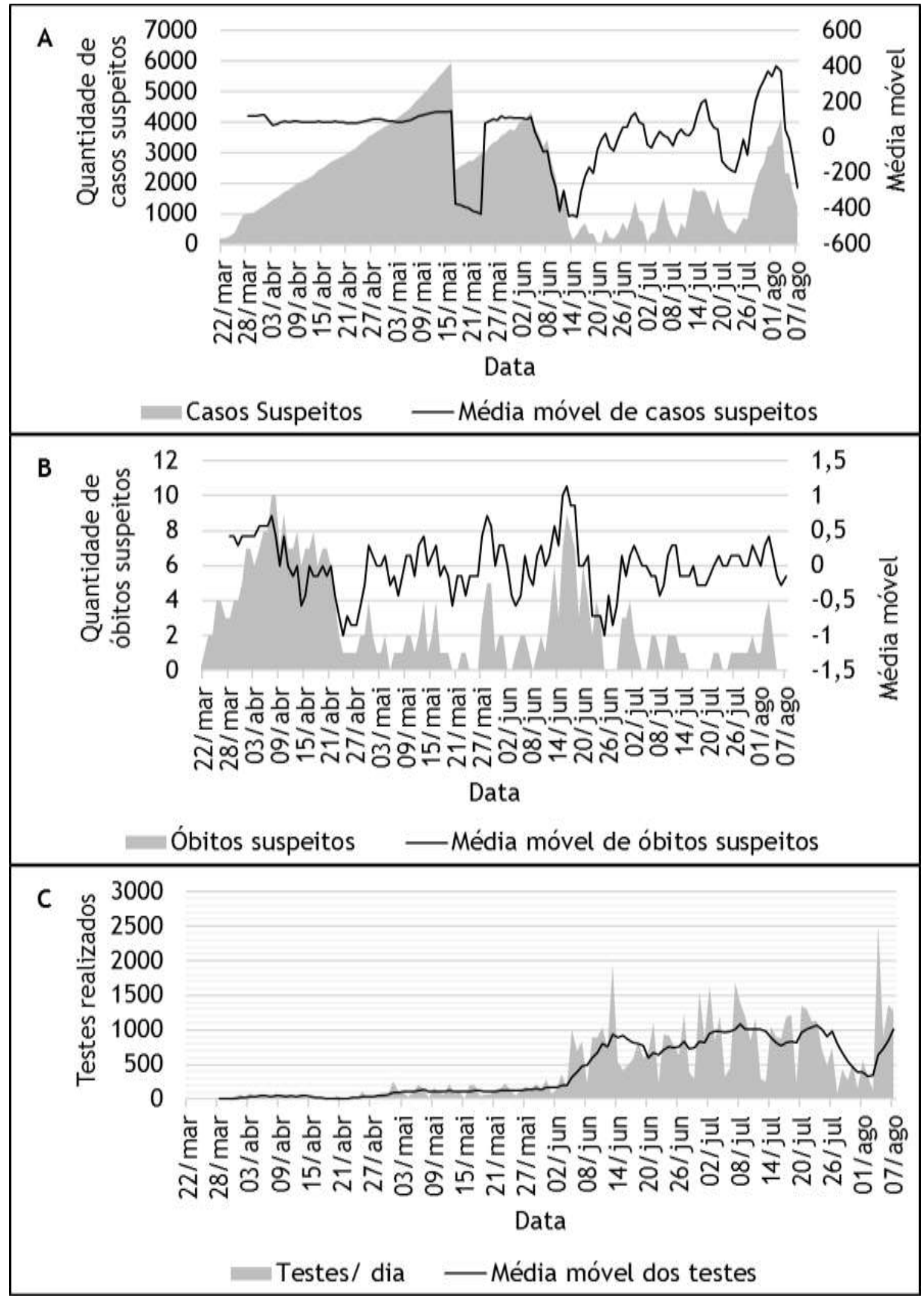

Figura 2 - A; Número e média móvel dos casos suspeitos da COVID-19; B: Número e média móvel dos óbitos suspeitos da COVID-19; C: Número de testes por dia e média móvel dos testes realizados para a COVID-19. Uberlândia (MG), Brasil, 2020. 
Tabela 1 - Análise da variação da média móvel do número de casos confirmados da COVID-19 a partir das datas de abertura e fechamento comercial em Uberlândia (MG), 2020.

\begin{tabular}{ccccc} 
Data & $\begin{array}{c}\text { Média } \\
\text { móvel } \\
\text { Inicial }\end{array}$ & $\begin{array}{c}\text { Média } \\
\text { móvel pós } \\
14 \text { dias }\end{array}$ & $\begin{array}{c}\text { Variação } \\
\text { percentual }\end{array}$ & Tendência \\
\hline $\begin{array}{c}15 \text { de abril: } \\
\text { abertura comercial, } \\
\text { exceto shoppings e } \\
\text { parques }\end{array}$ & 1,42 & 6,28 & $+342,25 \%$ & Crescimento \\
$\begin{array}{c}\text { 28 de maio: } \\
\text { abertura de shoppings } \\
\text { e parques }\end{array}$ & 49,28 & 172,71 & $+250,46 \%$ & Crescimento \\
$\begin{array}{c}19 \\
\text { 19 de junho: }\end{array}$ & 241,14 & 218,28 & $-9,47 \%$ & Estabilização \\
fechamento comercial & & & & \\
$\begin{array}{c}17 \text { de julho: } \\
\text { abertura comercial, } \\
\text { incluindo shoppings } \mathrm{p} \\
\text { parques }\end{array}$ & 162,42 & 95,28 & $-41,33 \%$ & Queda \\
\hline
\end{tabular}

Tabela 2 - Análise da variação percentual da média da taxa de crescimento em 24h dos casos confirmados da COVID-19, a partir das datas de abertura e fechamento comercial em Uberlândia (MG), 2020.

\begin{tabular}{|c|c|c|c|}
\hline Data & $\begin{array}{l}\text { Média da taxa de } \\
\text { crescimento inicial }\end{array}$ & $\begin{array}{c}\text { Média da taxa de } \\
\text { crescimento pós } \\
14 \text { dias }\end{array}$ & $\begin{array}{l}\text { Variação } \\
\text { percentual }\end{array}$ \\
\hline $\begin{array}{c}15 \text { de abril } \\
\text { abertura comercial, } \\
\text { exceto shoppings e } \\
\text { parques }\end{array}$ & 1,055 & 1,068 & $+1,23 \%$ \\
\hline $\begin{array}{c}28 \text { de maio } \\
\text { abertura de shoppings } \\
\text { e parques }\end{array}$ & 1,013 & 1,067 & $+5,33 \%$ \\
\hline $\begin{array}{l}19 \text { de junho } \\
\text { fechamento } \\
\text { comercial }\end{array}$ & 1,063 & 1,041 & - 2,06\% \\
\hline $\begin{array}{c}17 \text { de julho } \\
\text { abertura comercial, } \\
\text { incluindo shoppings e } \\
\text { parques }\end{array}$ & 1,025 & 1,018 & $-0,68 \%$ \\
\hline
\end{tabular}

Ocorreu expressivo aumento das internações pela COVID-19 nos leitos públicos e particulares do município para o período analisado. Maiores taxas de crescimento foram observadas nos primeiros meses da análise, com estabilização abaixo de 1,2 a partir de 18/06 e manutenção do número de pacientes internados entre 200 e 250 até o final do período analisado (Figura 3 A). Registrou-se maior quantidade de pacientes em leitos de enfermaria em relação aos internados em UTI (Figura 3 B). A média de alocação dos pacientes foi de $55 \%$ para enfermaria e de $45 \%$ para UTI. Houve predomínio relevante do sexo masculino entre os internados (Figura 3 - C). A média de alocação dos 
pacientes do sexo masculino foi de $49 \%$ e a de pacientes do sexo feminino de $41 \%$. Maiores de 60 anos somam mais da metade da média de alocação dos leitos (55\%) para as internações registradas. Pode-se observar maior frequência (29\%) de casos entre adultos de 40 a 59 anos quando comparados a outras faixas etárias isoladas, sendo a faixa etária pediátrica (4\%) e indivíduos de 13 a 39 anos $(11 \%)$ os que registraram menor número de internações (Figura 3 - D).

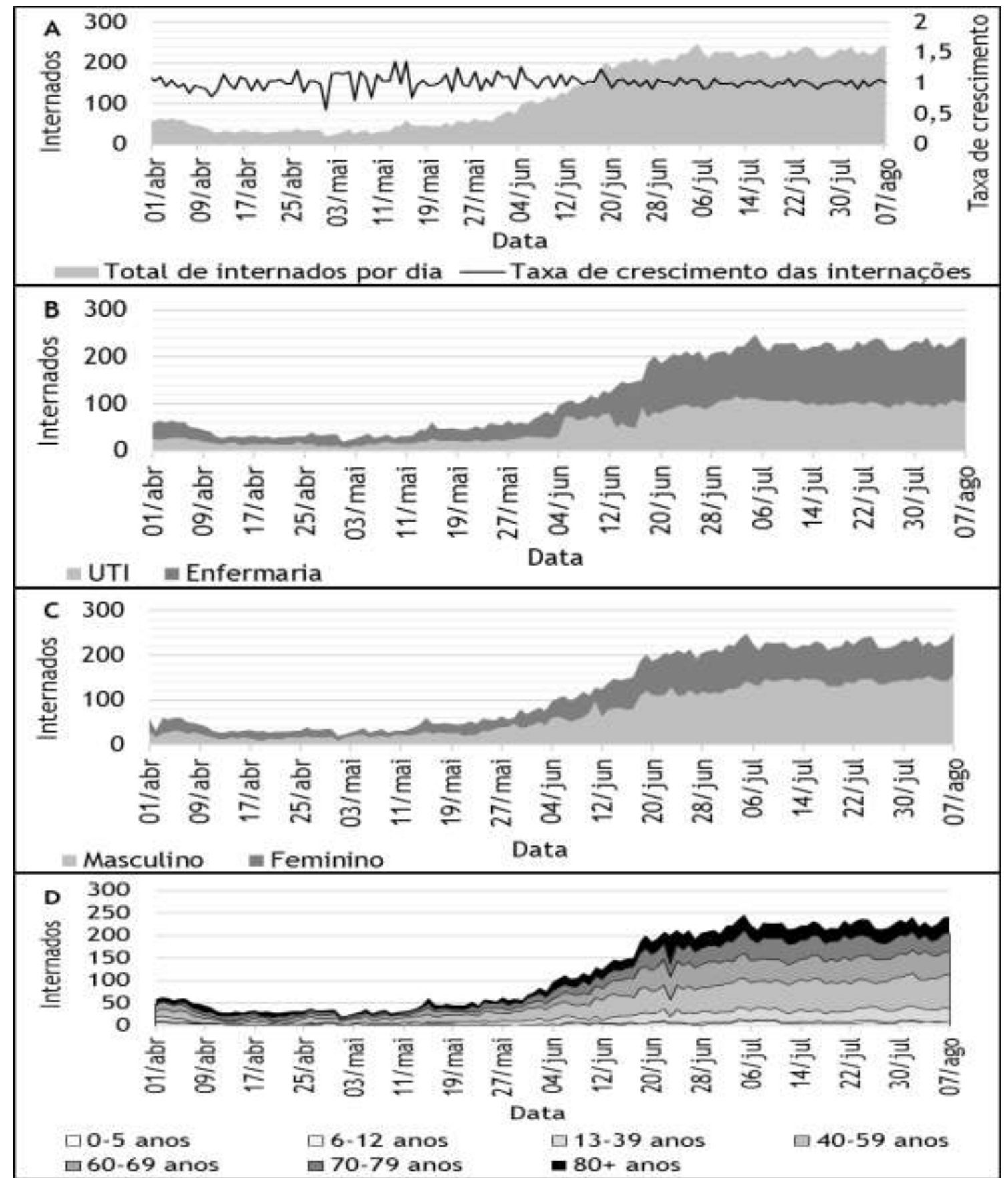

Figura 3 - A: Total de pacientes internados por dia e taxa de crescimento em 24 horas das internações pela COVID-19; B: Total de pacientes internados pela COVID-19 na rede pública e privada, por dia, segundo leito de ocupação; C: Total de pacientes internados pela COVID-19, segundo sexo biológico; D: Total de pacientes internados pela COVID-19, segundo faixa etária. Uberlândia (MG), Brasil, 2020. 


\section{DISCUSSÃO}

De acordo com os dados analisados, observou-se que Uberlândia segue com aumento expressivo do número de casos e óbitos confirmados. Também se nota expressiva incidência de casos confirmados, superior à incidência brasileira $(1.288,6 / 100 \mathrm{mil}$ habitantes), isto representa aproximadamente 790 casos a mais a cada 100 mil habitantes. Entretanto, quando comparadas as taxas de mortalidade e letalidade, Uberlândia mostrou valores inferiores aos nacionais (44,5 óbitos/100 mil habitantes e 3,45\% de letalidade). Apenas a Região Norte do país registrou incidência maior do que Uberlândia, a qual superou as regiões Sul e Centro-Oeste em taxa de mortalidade, sendo inferior ao restante das demais regiões $^{25}$.

A Região Sudeste, em relação ao município, registrou incidência inferior (1056,1 casos/100 mil habitantes) e taxa de mortalidade superior (47,8 óbitos/100 mil habitantes). Em Minas Gerais, incidência e taxa de mortalidade estaduais foram expressivamente menores do que os dados do município do triângulo mineiro: 614 casos/100 mil habitantes e 13,5 óbitos/100 mil habitantes, entretanto, com letalidade superior, de 2,1 óbitos/100 casos $^{25,26}$.

Segundo o boletim divulgado pela Secretaria de Estado de Saúde de Minas Gerais, Uberlândia possuía no dia 7 de agosto $9,06 \%$ do total de casos da unidade federativa e $7,48 \%$ do total de óbitos. Todavia, ressalta-se que para o dia analisado, o boletim epidemiológico estadual registrava 13.360 casos e 253 óbitos para o município uberlandense, uma subnotificação de 1.029 casos $(7,15 \%)$ e 12 óbitos $(4,52 \%)^{26}$. Nesse sentido, reitera-se que pode ocorrer atraso nas notificações tanto por sobrecarga laboratorial e lentificação da liberação de resultados de testes, como pela demora no compartilhamento dos dados entre diferentes instâncias, sendo, portanto, o atraso no compartilhamento de informações a causa provável da disparidade entre os números ${ }^{27}$. Durante pandemias, é essencial que haja celeridade na transmissão e divulgação dos dados epidemiológicos que sustentem as ações de vigilância em saúde. No entanto, em várias cidades/regiões do país o registro e a divulgação das informações são demorados, revelando a necessidade de reestruturação do sistema de notificações no país, que segundo a OMS, deve estar mais preparado para o 
surgimento de novas pandemias, que têm se tornado cada vez mais frequentes ${ }^{11,27}$. De certa forma, esse cenário também se reflete em Uberlândia e no Estado mineiro.

0 número de casos confirmados da COVID-19 em relação ao número total de testes foi superior $(23,98 \%)$ ao verificado em outros países que realizam testagem em massa da população. Na Coreia do Sul, a taxa de testes positivos foi de $0,9 \%$, enquanto na Islândia foi de 5,8\% e, na Austrália foi de 0,1\% ${ }^{28-31}$. Enquanto a testagem em massa passou a ser o padrão internacional, no Brasil se restringe apenas aos casos sintomáticos ${ }^{32}$, justificando a taxa de positividade elevada. Nesse contexto, esse município deixa de diagnosticar muitos casos, principalmente assintomáticos, uma grande porcentagem que chegou a 30,8\% em Wuhan e a $51,7 \%$ no Japão 33,34 .

Essa situação é preocupante, visto que a infecciosidade dos pacientes assintomáticos permanece elevada, semelhante ou mesmo igual à dos sintomáticos ${ }^{33,34}$. No Brasil, estima-se que apenas $7,8 \%$ dos casos confirmados para COVID-19 são notificados e no estado de Minas Gerais somente 20,1\%35. No país, apenas 4.110,47 indivíduos/100mil habitantes foram testados até nove de agosto de 2020, já no estado de Minas Gerais foi realizado um total de 469.586 testes, sendo 2.218,29 testes/100 mil habitantes. As taxas de testagem registradas país e no estado foram bem inferiores em relação a Uberlândia, de 8.678,36 testes/100 mil habitantes, concentrando $12,77 \%$ dos testes estaduais ${ }^{36}$.

Em Uberlândia, o aumento do número de casos confirmados passou a ocorrer após a segunda semana de maio, coincidindo juntamente com o fim do pico dos casos suspeitos, o que pode refletir o incremento na testagem da população, que antes passava por uma possível subnotificação. Esse contexto sugere que houve uma resposta morosa do município no enfrentamento ao coronavírus, visto que a liberação em tempo hábil dos resultados dos testes é essencial para 0 monitoramento epidemiológico ${ }^{11,27}$. Esse panorama condiz com o padrão encontrado no território brasileiro e pode ser atribuído ao desabastecimento de insumos para testagem, associado à falta de preparo dos profissionais, que acabaram por prejudicar as ações em Vigilância Epidemiológica em Saúde ${ }^{11,27}$. Dessa forma, o monitoramento das emergências, preconizado pelo SUS, fica comprometido, dificultando que haja 
planejamento racional das medidas a serem tomadas em direção ao manejo rápido da pandemia ${ }^{11,12,37}$.

Durante o período, é possível notar aumentos e quedas expressivos da média móvel de casos, e em menor quantidade de óbitos. As aberturas do comércio e de espaços de lazer de 15 de abril e 28 de maio precederam períodos de aumento das médias móveis e médias das taxas de crescimento de casos confirmados para a COVID-19, com tendência de crescimento confirmada. Enquanto a paralisação das atividades realizada a partir de 19 de junho precedeu um período de diminuição da média móvel e da taxa de crescimento dos casos confirmados, com padrão de estabilização devido à queda inferior a 15\%. Os óbitos confirmados se comportaram de forma semelhante, acompanhando os períodos de aumento ou queda percentuais. Entretanto, ambos os dados voltaram a subir mesmo durante 0 não funcionamento das atividades comerciais não essenciais. Apesar disso, segundo a literatura as medidas de distanciamento social ainda se mostram efetivas e de extrema importância no controle da pandemia, na redução de casos e óbitos e na prevenção do colapso dos sistemas de saúde locais ${ }^{32}$. Nesse sentido, a inexistência de dados que mensurem a taxa de distanciamento social em Uberlândia limita a análise adequada das medidas colocadas em prática pela prefeitura e se houve realmente adesão por parte da população aos decretos municipais. Segundo o Guia de Vigilância em Saúde do Ministério da Saúde ${ }^{11}$, a análise de condições que afetem a propagação da doença e as medidas de controle empregadas compõe a investigação de surtos e epidemias, sendo extremamente relevante, nesse contexto, monitoramento da taxa de distanciamento populacional, medida que, portanto, deveria ser adotada pelo munícipio uberlandense.

Contraditoriamente, a reabertura comercial de 17 de julho precedeu um período de redução da média da taxa de crescimento e da média móvel dos casos confirmados, com padrão de queda confirmado (redução superior a 15\%). Concomitantemente houve queda na quantidade de testes realizados diariamente, com acúmulo expressivo de casos suspeitos e manutenção de óbitos e internações. Esse padrão reflete uma possível subnotificação de casos, relacionada à queda na taxa de testagem populacional, reforçado pelo não acúmulo de óbitos suspeitos, talvez devido à prioridade 
diagnóstica dada aos casos mais graves e aos óbitos na rede pública, via método RT-PCR ${ }^{38}$. Em colaboração com esse cenário, destaca-se ainda a acurácia deficiente tanto do teste rápido, cujos índices de falso-negativo variam de $10 \mathrm{a}$ $44 \%^{37}$, como do próprio Reverse Transcriptase Polymerase Chain Reaction (RT-PCR), principalmente nos estágios iniciais da infecção ${ }^{39}$.

O que chama atenção na COVID19 são as altas taxas de alocação dos pacientes em UTI, correspondendo a $45 \%$ das internações diárias. Esse número elevado também reflete a concentração da testagem em indivíduos mais graves quando comparados com dados da OMS, os quais apontam que $15 \%$ dos pacientes com COVID-19 necessitam de hospitalização com oxigenoterapia e 5\% tem necessidade de internação em UTI ${ }^{40}$.

A ocupação dos leitos de UTI está vinculada à admissão por instabilidade crítica, com necessidade de intervenção de suporte à vida, sendo a prioridade para os pacientes com alta probabilidade de recuperação ${ }^{41}$. Embora ainda não seja o cenário ideal, Uberlândia está localizado na região Sudeste, que apresenta infraestrutura hospitalar mais adequada para lidar com a pandemia, quando comparado aos vazios assistenciais do Norte e Nordeste ${ }^{41}$.

Em consonância com a literatura, homens apresentaram maior número de internações diárias quando comparados às mulheres, o que pode estar relacionado tanto ao desenvolvimento de formas mais graves, quanto a maior exposição a situações de risco ${ }^{42,43}$. Esse contexto poder estar relacionado com o fato de a população masculina possuir maior taxa de comorbidades e maus hábitos de vida, como tabagismo e etilismo, além de serem mais resistentes às medidas sanitárias impostas, como o ato de lavar as mãos ou de realizar o isolamento social ${ }^{43}$.

Quanto à faixa etária, os idosos lideram as internações, principalmente, por serem o grupo de maior prevalência de comorbidades $^{44}$, incluindo cardiovasculares, associadas a um pior prognóstico da infecção ${ }^{43}$. Em contrapartida, os pacientes pediátricos possuem menor taxa de internação em relação aos adultos, assim como os estudos da literatura ${ }^{45}$.

Ressalta-se que a prática da vigilância em saúde adequada acompanhada de investigação epidemiológica efetiva durante epidemias é uma atividade obrigatória 
de todo sistema local de saúde, e deve ocorrer de forma integrada e concomitante à ações de prevenção e controle, com divulgação de dados com maior brevidade possível à serviços relacionados, à instâncias superiores, à sociedade e seus representantes ${ }^{25}$. De acordo com o Plano de Contingência Nacional para Infecção Humana pelo novo Coronavírus do Ministério da Saúde, cabe aos serviços de vigilância em saúde estabelecer estratégias de comunicação de risco, com levantamento e divulgação de informações que ajudem no combate e no controle à doença ${ }^{46}$. Frente a isso, evidencia-se a necessidade de intervenções contínuas de vigilância em saúde $^{47}$ em tempos de pandemia, acompanhada da melhora dos sistemas de notificação e repasse de informações entre diferentes instâncias, e de investimentos em estratégias de testagem da população, associadas a políticas de distanciamento social monitoradas, com levantamento da taxa de isolamento social local, visto que, como observado no presente estudo, decretos municipais de fechamento comercial, sem a vigilância adequada, podem não ser suficientes no controle de casos, óbitos e internações.

O presente estudo se limita aos dados de divulgação pela Secretaria
Municipal do Município de Uberlândia, que traz apenas os casos separados por sexo, faixa etária ou tipo de leito ocupado por dia e não sua totalidade desde o início dos registros dos dados, o que restringe a análise, uma vez que um mesmo indivíduo pode ficar internado por vários dias. Esse panorama dificulta a obtenção de informações mais precisas pelo restante dos serviços da cidade e, consequentemente, a atuação conjunta dos mesmos no controle da pandemia.

\section{CONCLUSÃO}

Foram analisados os dados epidemiológicos da COVID-19 em Uberlândia e discutido o impacto das medidas adotadas pela prefeitura municipal, percebendo expressiva influência do grau de abertura comercial nos indicadores da doença. Assim como no município analisado, outras localidades do país estão sujeitas à baixa disponibilidade de testes, subnotificação expressiva e falta de informações adequadas e organizadas a respeito do controle da pandemia.

Cabe destacar também a expressiva influência do grau de abertura comercial no incremento da frequência de testes positivos, casos e óbitos, com importante manutenção de 
casos em enfermarias e UTI, o que aponta para a necessidade de aumento da eficácia das ações de controle da pandemia a nível local. Por isso, análises como esta devem ser encorajadas e estimuladas, fornecendo mais dados a respeito do combate à doença no Brasil, de maneira a expandir a troca de informações e melhorar a eficácia das ações de vigilância em saúde.

\section{REFERÊNCIAS}

1. WHO. Coronavirus Disease (COVID-19) Pneumonia of unknown cause - China [internet]. World Health Organization; 2020 [citado em 10 ago 2020]. Disponível em: https://www.who.int/csr/don/05january-2020-pneumonia-of-unkowncause-china/en/.

2. WHO. Coronavirus Disease (COVID-19) Novel Coronavirus - China [internet]. World Health Organization; 2020 [citado em 10 ago 2020]. Disponível em:

https: / /www.who.int/csr/don/12january-2020-novel-coronaviruschina/en/.

3. WHO. Coronavirus Disease (COVID-19) Statement on the second meeting of the International Health Regulations (2005) Emergency Committee regarding the outbreak of novel coronavirus (2019-nCoV) [internet]. World Health Organization; 2020 [citado em 10 ago 2020]. Disponível em: https://www.who.int/newsroom/detail/30-01-2020-statementon-the-second-meeting-of-theinternational-health-regulations(2005)-emergency-committeeregarding-the-outbreak-of-novelcoronavirus-(2019-ncov).

4. Croda JHR, Garcia LP. Resposta imediata da Vigilância em Saúde à epidemia da COVID-19. Epidemiol Serv Saúde. 2020; 29(1):e2020002.

5. Rafael RMS, Neto M, Carvalho MMB, David HMSL, Acioli S, Faria MGA. Epidemiologia, políticas públicas e pandemia de Covid-19: o que esperar no Brasil? Rev enferm UERJ. 2020; 28(1):e49570.

6. Wu JT, Leung $\mathrm{K}$, Leung GM. Nowcasting and forecasting the potential domestic and international spread of the 2019-nCoV outbreak originating in Wuhan, China: a modelling study. The Lancet. 2020; 395(10225):689-697.

7. Qun L, Guan X, Wu P, Wang X, Zhou L, Tong $Y$, et al. Early Transmission Dynamics in Wuhan, China, of Novel Coronavirus- Infected Pneumonia. N Engl J Med. 2020; 382:1199-1207. 
8. Farias HS. O avanço da Covid-19 e o isolamento social como estratégia para redução da vulnerabilidade. Espaço e Econ. 2020; 17:1-12.

9. Nicola M, Alsafi Z, Sohrabi C, Kerwan A, Al-Jabir A, losifidis C, et al. The socio-economic implications of the coronavirus pandemic (COVID-19): A review. Int J Surg. 2020; 78:185-193.

10. Bong CL, Brasher C, Chikumba E, McDougall R, Mellin-Olsen J, Enright A. The COVID-19 Pandemic: Effects on Low- and Middle-Income Countries. Anesth Analg. 2020; 131(1):86-92.

11. Ministério da Saúde (BR), Secretaria de Vigilância Epidemiológica, Departamento de Vigilância Epidemiológica. Guia de Vigilância em Saúde. Brasília, DF: Ministério da Saúde; 2019

12. Conselho Nacional de Secretários de Saúde- Conass (BR). Vigilância em Saúde - Parte 1. Conselho Nacional de Secretários de Saúde. Brasília, DF: Conass, 2011.

13. Prefeitura Municipal de Uberlândia. Diário Oficial do Município No 5817. Prefeitura de Uberlândia; 2020 [citado em 10 Ago 2020]. Disponível em:

http: //docs.uberlandia.mg.gov.br/w pcontent/uploads/2020/02/5817.pdf \#page=02

14. Secretaria de Estado de Saúde de Minas Gerais. Informe Epidemiológico Coronavírus 10/08/2020. Secretaria de Estado de saúde de Minas Gerais; 2020 [citado em 10 ago 2020]. Disponível em: https: //www.saude.mg.gov.br/coro navirus/boletim

15. Ministério da Saúde (BR). Diagnóstico Clínico e Laboratorial [internet]. Ministério da Saúde; 2020 [citado em 10 ago 2020]. Disponível em: https: //coronavirus. saude.gov.br/di agnostico-clinico-e-laboratorial

16. Cidades [internet]. Brasília: Ministério da Economia, Instituto Brasileiro de Geografia e Estatística; [citado em 2020 Ago 10]. Disponível em:

https: //cidades.ibge.gov.br/brasil/ $\mathrm{mg} /$ panorama

17. Prefeitura Municipal de Uberlândia. Comitê Municipal de Enfrentamento ao COVID-19. Prefeitura de Uberlândia; 2020 [citado em 10 Ago 2020]. Disponível em: http://docs.uberlandia.mg.gov.br/w p-

content/uploads/2020/04/Delibera\% C3\%A7\%C3\%A3o-Comit\%C3\%AACOVID-19-15.04.2020.pdf 
18. Prefeitura Municipal de Uberlândia. Comitê Municipal de Enfrentamento ao COVID-19. Prefeitura de Uberlândia; 2020 [citado em 10 Ago 2020]. Disponível em: http://docs.uberlandia.mg.gov.br/w p-

content/uploads/2020/05/Delibera\% C3\%A7\%C3\%A30-006_2020-NE-Auto-

Escolas-e-Clubes-de-Lazer.pdf

19. Prefeitura Municipal de Uberlândia. Comitê Municipal de Enfrentamento ao COVID-19. Prefeitura de Uberlândia; 2020 [citado em 10 Ago 2020]. Disponível em: http://docs.uberlandia.mg.gov.br/w $\mathrm{p}-$

content/uploads/2020/06/Delibera\% C3\%A7\%C3\%A30-010_2020-NE.pdf

20. Prefeitura Municipal de Uberlândia. Comitê Municipal de Enfrentamento ao COVID-19. Prefeitura de Uberlândia; 2020 [citado em 10 Ago 2020]. Disponível em: http://docs.uberlandia.mg.gov.br/w p-

content/uploads/2020/07/DELIBERA \%С3\%87\%C3\%830-014-17.07.2020NE.pdf

21. Chowell G. Fitting dynamic models to epidemic outbreaks with quantified uncertainty: A Primer for parameter uncertainty, identifiability, and forecasts. Infect Dis Model. 2017;2(3):379-398.

22. Roncalli A, Lacerda JS. Jornalismo como forma de conhecimento: a questão da divergência dos dados de tendência da covid-19 divulgadospelo consórcio de imprensa e pela SESAP-RN. Scielo preprints; 2020 [citado em 6 Set 2020] Disponível em: https: //preprints.scielo.org/index.p $\mathrm{hp} / \mathrm{scielo} /$ preprint/view/1141/1712.

23. Henriques CMP, Vasconcelos W. Crises dentro da crise: respostas, incertezas e desencontros no combate à pandemia da Covid-19 no Brasil. Estud av. 2020;34(99):25-44.

24. Ministério da Saúde (BR). Conselho Nacional de Saúde. Resolução $n^{\circ}$. 510, de 07 de abril de 2016. Diário Oficial da União 2016 Mai. 24; Seção 1. p. 44-46.

25. Secretaria de Vigilância em Saúde. Boletim Epidemiológico Especial Semana Epidemiológica 31. Secretaria de Vigilância em Saúde; 2020 [citado em 10 ago 2020]. Disponível em: https: //saude.gov.br/images/pdf/20 20/August/06/Boletimepidemiologico-COVID-25-final--1.pdf 
26. Centro de Operaçõos de Emergência em Saúde-COES Minas. Boletim Epidemiológico COVID-19: Doença causada pelo coronavirus. Centro de Operações de Emergência em SaúdeCOES Minas; 2020 [citado em 10 ago 2020]. Disponível em: http: //coronavirus.saude.mg.gov.br /images/boletim/07-08Boletim_Epidemiologico_COVID19.pdf

27. Lana RM, Coelho FC, Gomes MFDC, Cruz OG, Bastos LS, Villela DAM, et al. Emergência do novo coronavírus (SARS-CoV-2) e o papel de uma vigilância nacional em saúde oportuna e efetiva. Cad Saúde Pública. 2020; 36(3):e00019620.

28. Hallal PC, Horta BL, Barros AJD, Dellagostin AO, Hartwig FP, Pellanda LC, et al. Evolução da prevalência de infecção por COVID-19 no Rio Grande do Sul, Brasil: inquéritos sorológicos seriados. Ciênc Saúde Coletiva. 2020;25(1):2395-2401.

29.Gudbjartsson DF, Helgason A, Johsson $\mathrm{H}$, Magnusson OT, Melsted P, Norddahl GL, et al. Early Spread of SARS-Cov-2 in Icelandic Population [internet]. medRxiv. 2020. [citado em 10 ago 2020]. Disponível em: https://www.medrxiv.org/content/1 $0.1101 / 2020.03 .26 .20044446 v 2$
30. Central Disease Control Headquarters. Coronavirus Disease19, Republic of Korea. Central Disease Control Headquarters; 2020 [citado em 10 ago 2020]. Disponível em: http://ncov.mohw.go.kr/en

31. Australian Government Deparment of Health. COVID-19, Australia: Epidemiology Report 18: Fortnightly reporting period ending 7 June 2020. Commun Dis Intell. 2020; 44(1).

32. Ministério da Saúde (Brasil). Sobre a doença [internet]. Ministério da Saúde; 2020 [citado em 10 ago 2020]. Disponível em: https: / / coronavirus. saude.gov.br/so bre-a-doenca

33. Gao Z, Xu Y, Sun C, Wang X, Guo Y, Qiu $S$, et al. A Systematic Review of Asymptomatic Infections with COVID19. J Microbiol Immunol Infect. 2020.

34. Tan J, Liu S, Zhuang L, Chen L, Dong M, Zhang J, et al. Transmission and clinical characteristics of asymptomatic patients with SARSCoV-2 infection. Future Virol. 2020.

35. Prado MF, Antunes BBP, Bastos, LSL, Peres IT, Silva AAB, Dantas LF, et al. Análise da subnotificação de COVID19 no Brasil. Rev Bras Ter Intensiva. 2020; 32(2):224-228. 
36. Cota W. Monitoring the number of COVID-19 cases and deaths in Brazil at municipal and federative units level. Scientific Eletronic Library Online. 2020. [citado em 10 ago 2020]. Disponível em: https: / /covid19br.wcota.me/

37. Castro R, Luz PM, Wakimoto MD, Veloso VG, Grinsztejn B, Perazzo $\mathrm{H}$. COVID-19: a meta-analysis of diagnostic test accuracy of comercial assays registered in Brazil. Brazil J Infect Dis. 2020; 24(2):180-187.

38. Ministério da Saúde (Brasil). Saúde passa a testar casos leves de Covid19 [internet]. Ministério da Saúde; 2020 [citado em 10 ago 2020]. Disponível em: https://www.saude.gov.br/noticias/ agencia-saude/47113-saude-passa-atestar-100-dos-casos-leves-de-covid19

39. Kucrika LM, Lauer SF, Laeyendecker O, Boon D. Variation in FalseNegativa Rate of Reverse Transcriptase Polymerase Chain Reaction-Based SARS-CoV-2 Tests by Time Since Exposure.Ann Intern Med 2020.

40. Noronha KVMS, Guedes GR, Turra CM, Andrade MV, Botega L, Nogueira D, et al. Pandemia por COVID-19 no Brasil: análise da demanda e da oferta de leitos hospitalares e equipamentos de ventilação assistida segundo diferentes cenários. Cad Saúde Pública. 2020;36(6):e00115320.

41. Moreira RS. COVID-19: unidades de terapia intensiva, ventiladores mecânicos e perfis latentes de mortalidade associados à letalidade no Brasil. Cad Saúde Pública. 2020; 36(5):e00080020.

42. Galbadage T, Peterson BM, Awada J, Buck AS, Ramirez DA, Wilson J, et al. Systematic Review and Meta-Analysis of Sex-Specific COVID-19 Clinical Outcomes. Front Med (Lausanne). 2020; 7:348.

43. Gebhard C, Regitz-Zagrosek V, Neuhauser HK, Morgan R, Klein SL. Impact of sex and gender on COVID19 outcomes in Europe.Biol Sex Differ. 2020; 11(1).

44. Cockerham WC, Hamby BW, Oates GR. The Social Determinants of Chronic Disease. Am J Prev Med. 2017; 52(1):S5-S12.

45. Brodin P. Why is COVID-19 so mild in children? Acta Paediatr. 2020; 109(6):1082-1083.

46. Ministério da Saúde (BR). Centro de Operações de Emergências em Saúde Pública. Plano de Contingência Nacional para Infecção Humana pelo 
novo Coronavírus COVID-19. Brasília, DF: Ministério da Saúde; 2020.

47. Mendonça FD, Rocha SS, Pinheiro DLP, Oliveira SV. Região Norte do
Brasil e a pandemia de COVID-19: análise socioeconômica e epidemiológica. J Health NPEPS. 2020; 5(1):20-37.

Financiamento: Os autores declaram que não houve financiamento.

Conflito de interesses: Os autores declaram não haver conflito de interesses.

\section{Participação dos autores:}

- Concepção: Rosa MFP, Silva WNT, Carvalho WRG, Oliveira SV.

- Desenvolvimento: Rosa MFP, Silva WNT, Carvalho WRG, Oliveira SV.

- Redação e revisão: Rosa MFP, Silva WNT, Carvalho WRG, Oliveira SV.

Como citar este artigo: Rosa MFP, Silva WNT, Carvalho WRG, Oliveira SV. Epidemiologia da COVID-19 em Uberlândia (MG): análise preliminar do impacto do grau de abertura comercial. J Health NPEPS. 2020; 5(2):20-41. 